\title{
Arco Axilar de Langer (Músculo Axilopectoral): Variante Supernumeraria Inusual del Músculo Latísimo del Dorso. Reporte de Tres Casos
}

\author{
Langers's Axillary Arch (Axillopectoral Muscle): Inusual \\ Supernumerary Variant's Latissimus Dorsi. Three Cases Report \\ "Jesús Ignacio Ortiz; **Fernando Ramirez V.; **Pierina Petrosino; \\ ${ }^{* * *}$ Melisse Milano; ${ }^{* *}$ Asmiria Arenas \& ${ }^{* * *}$ Virgilio Castillo
}

ORTIZ, J. I.; RAMIREZ, V. F.; PETROSino, P.; MilANO, M.; ARENAS, A. \& CASTILLO, V. Arco axilar de Langer (músculo axilopectoral): variante supernumeraria inusual del músculo latísimo del dorso. Reporte de tres casos. Int. J. Morphol., 27(4):12091212, 2009.

RESUMEN: El arco axilar de Langer o músculo axilopectoral es una variación relativamente rara de la inserción del músculo latísimo del dorso. Se identifica en aproximadamente el 1.7 a $7 \%$ de las disecciones axilares y corresponde a un complejo músculofascial, la parte muscular junto con el tendón del músculo pectoral mayor se inserta dentro del labio lateral del surco intertubercular del húmero, mientras que, la parte fascial está formada por bandas fibrosas que se extienden en la parte profunda del músculo pectoral mayor para insertarse en el proceso coracoide entre la unión de los músculos coracobraquial y pectoral menor. El reconocimiento de esta anomalía es importante para los médicos clínicos, cirujanos y fisioterapeutas, ya que obliga a plantearse el diagnóstico diferencial de masas axilares, historia de obstrucción venosa axilar intermitente, o su aparición inesperada en el transcurso de la disección axilar del linfonodo centinela o linfadenectomías. Se presentan tres casos de arco axilar de Langer diagnosticados en la Unidad de Patología Mamaria del Instituto Autónomo Hospital Universitario de los Andes, Mérida, Venezuela, en el periodo 1999-2006, en el transcurso de 210 disecciones axilares practicadas en pacientes con cáncer de mama para identificación del linfonodo centinela o en linfadenectomías.

PALABRAS CLAVE: Arco axilar de Langer; Músculo axilopectoral.

\section{INTRODUCCIÓN}

Entre las variaciones anatómicas relativamente raras del músculo latísimo del dorso se describe el arco axilar de Langer o músculo axilopectoral que corresponde a una variante de la inserción del músculo latísimo del dorso (Williams \& Warwick, 1985; Keshtgar et al., 1999; Bonastre et al., 2002). Este es identificado en aproximadamente el 1,7 y $7 \%$ de las disecciones axilares (Besana-Ciani \& Greenall, 2005; Chêne et al., 2007). Generalmente este músculo supernumerario nace del borde del latísimo del dorso. En el arco axilar hay un complejo musculo fascial continuo con las fibras del músculo latísimo del dorso. La parte muscular, junto con el tendón del músculo pectoral mayor se inserta dentro del labio del surco intertubercular del húmero, mientras que la parte fascial está formada por bandas fibrosas que se extienden profundamente desde el músculo pectoral mayor hasta insertarse en el proceso coracoides, en la parte media del pliegue posterior de la axila y cruza la axila por delante de los vasos y nervios axilares para unirse con la cara inferior del tendón del músculo pectoral mayor, al músculo coracobraquial o a la fascia del músculo bíceps braquial, y varía entre 7 a $10 \mathrm{~cm}$ de longitud por 0.5 a $2 \mathrm{~cm}$ de ancho (Williams \& Warwick). Es importante para el cirujano reconocerlo y escindirlo para exponer la arteria y vena axilares en pacientes con traumatismos y para la linfadenopatía o bypass axilar. Además, debe ser considerado en el diagnóstico diferencial de masas axilares o historia de obstrucción venosa axilar intermitente (Miguel et al., 2001). Igualmente, los fisioterapeutas deben reconocer la presencia del arco

* Unidad de Patología Mamaria, Universidad de Los Andes. Mérida, Venezuela.

** Departamento de Anatomía Patológica, Universidad de Los Andes, Mérida. Venezuela.

*** Departamento de Anatomía Humana, Universidad de Los Andes, Mérida, Venezuela. 
axilar de Langer ya que se presume que puede ocasionar compresión de vasos axilares y de los nervios musculocutaneo, mediano y ulnar durante la abducción pasiva/rotación lateral del hombro (Smith \& Cummings, 2006). Si la presencia de este músculo conlleva a trastornos clínicos o aparece inesperadamente en la disección quirúrgica de la axila (linfadenectomía o exploración del linfonofo centinela) la escisión del mismo es necesaria (Miguel et al.; Smith \& Cummings; Sachatello, 1977).

\section{CASOS CLÍNICOS}

Caso Clínico 1. Paciente género femenino de 37 años de edad, quien consulta por nódulo de mama derecha. Antecedentes familiares: Madre fallecida por cáncer (CA) de mama. Niega antecedentes personales de relevancia. Al examen físico de ingreso, paciente en buenas condiciones generales, cardiopulmonar estable. A la exploración de la mama derecha se palpa nódulo de $2.5 \mathrm{~cm}$ de diámetro, localizado a nivel del cuadrante inferomedial, de consistencia firme y fijo. Axila derecha con nódulo de $1 \mathrm{~cm}$ de diámetro. Resto de la exploración física sin cambios relevantes. Bajo la sospecha clínica de cáncer de mama se solicita mamografía, ecografía mamaria y se práctica punción aspiración con aguja fina (PAAF), cuyos resultados orientaron a neoplasia maligna de la mama. Se toma biopsia trucore para estudio histológico que concluye como carcinoma ductal infiltrante moderadamente diferenciado de mama derecha. Con el diagnóstico clínico de cáncer de Mama derecha cT2N1 Mo Estadio IIb, se decide mastectomía radical preservadora. En el tiempo axilar de la intervención quirúrgica se identificó un arco muscular en el hueco axilar, hacia lateral que procedía del músculo latísimo del dorso y se dirigía hacia el brazo recubriendo la vena axilar, el cual fue respetado (Fig. 1).

Caso Clínico 2. Paciente género femenino de 41 años de edad, quien es referida a la Sección de Patología Mamaria del Instituto Autónomo Hospital Universitario de los Andes por presentar tumoración en mama izquierda de tres meses de evolución. Niega antecedentes familiares o personales de importancia. A la exploración física sólo destaca la presencia de masa tumoral a nivel de la unión de los cuadrantes laterales de la mama izquierda, de $3 \mathrm{~cm}$ de diámetro, no móvil y de consistencia firme. Los estudios por imágenes (mamografía y ecografía mamaria, concluyen como neoplasia maligna. La PAAF informa positividad para malignidad y la biopsia trucore reporta carcinoma ductal infiltrante moderadamente diferenciado de mama izquierda. El caso se discute en clínica de tumores y con diagnóstico clínico de cáncer de mama izquierda pT2N1(gc)Mo estadio Iib se decide tratamiento quirúrgico.

Fue sometida a identificación y biopsia con azul patente del linfonodo centinela axilar izquierdo. La biopsia por congelación del linfonodo centinela reportó positivo para metástasis. Inmediatamente se procedió a realizar una disección nodular axilar total. Durante la disección nodular axilar al separar el tejido linfocelulonodular del borde inferior de la vena axilar en dirección hacia el brazo, recubriendo parcialmente el paquete vascular escapular y por fuera de este, se identificó y respetó un arco muscular grueso que recubría la vena axilar, con inserciones entre el músculo latísimo del dorso por abajo, y por arriba pasaba por debajo del músculo pectoral mayor hacia el surco intertubercular, el cual se respetó.

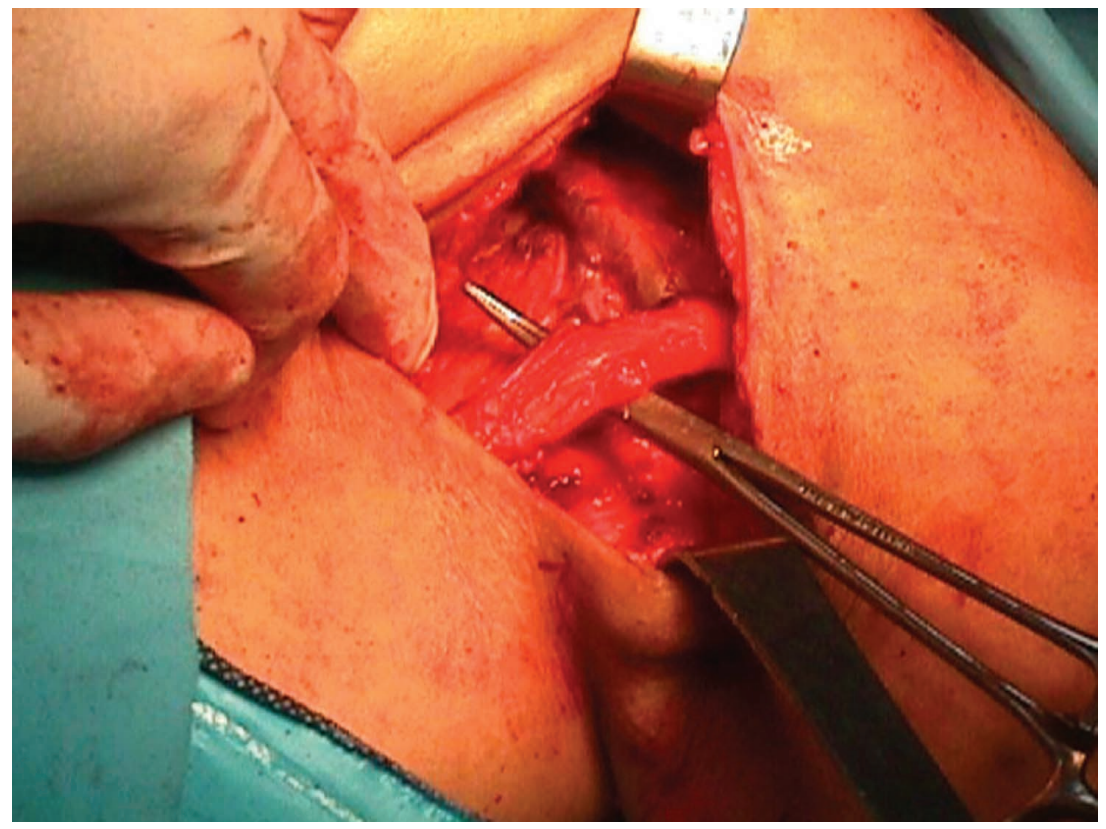

Fig. 1. Arco axilar de Langer. 
Caso clínico 3. Paciente género femenino de 43 años de edad, quien acude a consulta por presentar lesión nodular de mama izquierda. Antecedentes familiares y personales no relevantes. En el examen físico de ingreso sólo destaca como hallazgo de importancia la presencia de masa tumoral localizada a nivel de cuadrante superolateral izquierdo, de 3 $\mathrm{cm}$ de diámetro, firme, fijo y a nivel de la piel suprayacente aspecto de "corteza o piel de naranja". Posterior a los resultados positivos de la mamografía, ecografía mamaria y PAFF, se toma biopsia trucore que reporta carcinoma intraductal en un $90 \%$ con áreas de micro invasión y de comedocarcinoma de mama izquierda. Se planifica identificación y biopsia del linfonodo centinela axilar izquierdo con azul patente, el cual fue informado por corte congelado como positivo para micro metástasis. Se procedió a realizar disección nodular axilar durante la cual se identificó un arco muscular en el hueco axilar hacia lateral que procedía del músculo latísimo del dorso y se dirigía hacia el brazo recubriendo la vena axilar. Este arco muscular fue resecado en bloque con el paquete linfocelulonodular axilar correspondiente a la disección axilar. La paciente no manifestó durante el seguimiento limitaciones funcionales durante su rehabilitación.

\section{DISCUSIÓN}

El arco axilar de Langer o músculo axilopectoral es una variación relativamente rara de la inserción del músculo latísimo del dorso. Se identifica en aproximadamente el 1,7 a $7 \%$ de las disecciones axilares. El músculo latísimo del dorso es un músculo grande, triangular y plano, extendi- do sobre la región lumbar y mitad inferior del tórax, desde donde su fibras convergen hacia un estrecho tendón (Williams \& Warwick).

Los cirujanos deben estar advertidos de la eventual presencia del arco axilar de Langerd. El reconocimiento preoperatorio de esta anomalía posiblemente se puede evidenciarse por flebografía, por pérdida anormal de la concavidad axilar o por la marcada disparidad entre la axila visualmente llena y la dificultad encontrada en la palpación de masas axilares (Sachatello), y por resonancia magnética (Suzuna et al., 2003). Si la presencia de este músculo conlleva a trastornos clínicos o aparece inesperadamente en la disección quirúrgica de la axila (linfadenectomía o exploración del linfonofo centinela) la escisión del mismo es necesaria (Miguel et al.; Smith \& Cummings; Sachatello)

Esta variación muscular puede estar asociada a manifestaciones clínicas relacionadas con la compresión del paquete vasculonervioso axilar. Su reconocimiento es importante tanto para el diagnóstico diferencial de masa axilares, como para el abordaje quirúrgico de la axila en situaciones postraumáticas, linfadenopatías o bypass axilar.

El arco axilar de Langer o músculo axilopectoral es una entidad infrecuente ya que de 210 disecciones axilares practicadas en pacientes con cáncer de mama para identificación del linfonodo centinela o en linfadenectomías en la Unidad de Patología Mamaria del Instituto Autónomo Hospital Universitario de los Andes, Mérida, Venezuela, en el periodo 1999-2006, solamente se reportan estos tres casos, lo que representa una frecuencia de $1,4 \%$.

ORTIZ, J. I.; RAMIREZ, V. F.; PETROSINO, P.; MILANO, M.; ARENAS, A. \& CASTILLO, V. Langers's axillary arch (axillopectoral muscle): inusual supernumerary variant's latissimus dorsi. Three cases report. Int. J. Morphol., 27(4):1209-1212, 2009.

SUMMARY: The Langer's axillary arch or axillopectoral muscle is a is a relatively rare anatomical variation of latissimus dorsi muscle insertion. Langer's arch is identified in up to 1.7 a $7 \%$ of axillary explorations, and is a musclefascial complex continuous with the iliacal fibers of the latissimus dorsi. The muscular part, together with the tendon of pectoralis major muscle, inserted into the lateral lip of the intertubercular sulcus of the humerus, whereas the fascial part was formed by a fibrous band that extended deep to the pectoralis major muscle to insert into the coracoid process between the attachments of the coracobrachialis and pectoralis minor muscles. The recognition of this anomalies is important to clinicians, surgeons and physical therapist because It should be considered in the differential diagnosis of axillary masses an unexpected finding during axillary dissection by sentinel lymoh nodes or lymphadenectomy. We report three cases of Langer's axillary arch encountered during sentinel lymph node biopsy or lymphadenectomy en 210 patients with breast cancer, at the patology mammary Unit of the University Los Andes Hospital, in 1999-2006 period.

KEY WORDS: Langer's axillary arch; Axillopectoral muscle.

\section{REFERENCIAS BIBLIOGRÁFICAS}

Besana-Ciani, I. \& Greenall, M. J. Langer's axillary arch:

anatomy, embryological features and surgical implications. Surgeon, 3(5):325-7, 2005. 
ORTIZ, J. I.; RAMIREZ, V. F.; PETROSINO, P.; MILANO, M.; ARENAS, A. \& CASTILLO, V. Arco axilar de Langer (músculo axilopectoral): variante supernumeraria inusual del músculo latísimo del dorso. Reporte de tres casos. Int. J. Morphol., 27(4):1209-1212, 2009.

Bonastre, V.; Rodriguez- Niedenführ, M.; Choi, D. \& Sañudo, J. Coexistence of a pectoralis quartus muscle and an unusual axillary arch: case report and review. Clin. Anat., 15(5):366-70, 2002.

Chêne, G.; Le Bouëdec, G. \& Dauplat, J. Arch and sentinel: surgical technique of sentinel node biopsy with the axillopectoral muscle. Gynecol. Obst. Fertil., 35(1):259, 2007.

Keshtgar, M. R.; Saunders, C.; Ell, P. J. \& Baum, M. Langer's axillary arch in association with sentinel lymph node. Breast, 8(3):152-3, 1999.

Miguel, M.; Llusá, M.; Ortiz, J. C.; Porta, N.; Lorente, M. \& Götzens, V. The axillopectoral muscle (of Langer): report of three cases. Surg. Radiol. Anat., 23(5):341-3, 2001.

Smith, R. A. Jr. \& Cummings, J. P. The axillary arch: anatomy and suggested clinical manifestations. J. Orthop. Sports Phys. Ther, 36(6):425-9, 2006.

Sachatello, C. R. The axillopectoral muscle (Langer's axillary arch): a cause of axillary vein obstruction. Surgery, 81(5):610-2, 1977.

Suzuna, T.; Sacurai, T.; Yohimura, G.; Umemura, T.; Shimizu, Y.; Yang, Q. F. \& Okamura, Y. Magnetic resonance axillography for preoperative diagnosis of the axillopectoral muscle (Langer's axillary arch): a case report. Breast Cancer, 10(3):281-3, 2003.

Williams, P. L. \& Warwick, R. Gray's. Anatomía. 36a ed. Barcelona, Salvat, 1985. pp.623-4.
Dirección para correspondencia:

Jesús Ignacio Ortiz

Unidad de Patología Mamaria.

Facultad de Medicina

Universidad de Los Andes.

Mérida

VENEZUELA

Email: nachocancer@yahoo.mx

Recibido : 05-01-2009

Aceptado: 09-08-2009 